\section{Anti-monotone Constraints}

Carson K. Leung

Department of Computer Science, University of Manitoba, Winnipeg, MB, Canada

\section{Synonyms}

Anti-monotonic constraints

\section{Definition}

A constraint $C$ is anti-monotone if and only if for all itemsets $S$ and $S^{\prime}$ :

if $S \supseteq S^{\prime}$ and $S$ satisfies $C$, then $S^{\prime}$ satisfies $C$.

\section{Key Points}

Anti-monotone constraints [1, 2] possess the following nice property. If an itemset $S$ satisfies an anti-monotone constraint $C$, then all of its subsets also satisfy $C$ (i.e., $C$ is downward closed). Equivalently, any superset of an itemset violating an anti-monotone constraint $C$ also violates $C$. By exploiting this property, anti-monotone constraints can be used for pruning in frequent itemset mining with constraints. As frequent itemset mining with constraints aims to find itemsets that are frequent and satisfy the constraints, if an itemset violates an anti-monotone constraint $C$, all its supersets (which would also violate $C$ ) can be pruned away and their frequencies do not need to be counted. Examples of anti-monotone constraints include $\min ($ S.Price $) \geq \$ 20$ (which expresses that the minimum price of all items in an itemset $S$ is at least \$20) and the usual frequency constraint $\operatorname{support}(S) \geq \operatorname{minsup}$ (i.e., frequency $(S) \geq$ minsup). For the former, if the minimum price of all items in $S$ is less than $\$ 20$, adding more items to $S$ would not increase its minimum price (i.e., supersets of $S$ would not satisfy such an anti-monotone constraint). For the latter, it is widely used in frequent itemset mining, with or without constraints. It states that (i) all subsets of a frequent itemset are frequent and (ii) any superset of an infrequent itemset is also infrequent. This is also known as the Apriori property.

\section{Cross-References}

- Frequent Itemset Mining with Constraints

\section{Recommended Reading}

1. Lakshmanan LVS, Leung CK-S, Ng RT. Efficient dynamic mining of constrained frequent sets. ACM Trans Database Syst. 2003;28(4):337-89. doi:10.1145/958942.958944.

2. Ng RT, Lakshmanan LVS, Han J, Pang A. Exploratory mining and pruning optimizations of constrained associations rules. In: Proceedings of the ACM SIGMOD International Conference on Management of Data; 1998. p. 13-24. 